\title{
Predicting the minimum liquid surface tension activity of pseudomonads expressing biosurfactants
}

I. U. Mohammed, Y. Deeni, S. M. Hapca, K. McLaughlin and A. J. Spiers

This is the peer reviewed version of the following article:

Mohammed, I.U., et al. 2015. Predicting the minimum liquid surface tension activity of pseudomonads expressing biosurfactants. Letters in Applied Microbiology. 60(1): pp.37-43. Available from doi: 10.1111/ lam.12331

which has been published in final form at http://dx.doi.org/10.1111/ lam.12331. This article may be used for non-commercial purposes in accordance with Wiley Terms and Conditions for Self-Archiving. 
7 Original Article - Letters in Applied Microbiology

8

9 Predicting the minimum liquid surface tension activity of

10 pseudomonads expressing biosurfactants

11

I.U. Mohammed, Y. Deeni, S.M. Hapca, K. McLaughlin, \& A.J. Spiers

13

SIMBIOS Centre \& School of Science, Engineering and Technology, Abertay

University, Bell Street, DD1 1HG, Dundee, United Kingdom

\section{Correspondence}

Andrew Spiers, SIMBIOS Centre, Abertay University, Bell Street, DD1 1HG, Dundee, United Kingdom. E-mail: a.spiers@abertay.ac.uk

\section{Keywords}

Biosurfactant, Liquid surface tension, Pseudomonas, Surfactant

\section{Running Headline}

Surface tension activity of biosurfactants 


\section{SIGNIFICANCE AND IMPACT OF THE STUDY}

Numerous surveys of biosurfactant-producing bacteria have been conducted, but only recently has an attempt been made to predict the minimum liquid surface tension these surface-active agents can achieve. Here we determine a theoretical minimum of $24 \mathrm{mN}$ $\mathrm{m}^{-1}$ by statistical analysis of tensiometry data, suggesting a fundamental limit for biosurfactant activity in bacterial cultures incubated under standard growth conditions. This raises a challenge to our understanding of biosurfactant expression, secretion and function, as well as being of interest to biotechnology where they are used in an increasingly wide range of applications.

\section{ABSTRACT}

Bacteria produce a variety of biosurfactants capable of significantly reducing liquid (aqueous) surface tension $(\gamma)$ with a range of biological roles and biotechnological uses. In order to determine the lowest achievable surface tension $\left(\gamma_{\text {Min }}\right)$, we tested a diverse collection of Pseudomonas-like isolates from contaminated soil and activated sludge, and identified those expressing biosurfactants by drop-collapse assay. Liquid surface tension reducing ability was quantitatively determined by tensiometry, with 57 isolates found to significantly lower culture supernatant surface tensions to $24.5-49.1$ $\mathrm{mN} \mathrm{m}{ }^{-1}$. Differences in biosurfactant behaviour determined by foaming, emulsion and oil-displacement assays, was also observed amongst isolates producing surface tensions of $25-27 \mathrm{mN} \mathrm{m} \mathrm{m}^{-1}$, suggesting that a range of structurally-diverse biosurfactants were being expressed. Individual distribution identification (IDI) analysis was used to identify the theoretical probability distribution that best fitted the surface tension data, which predicted a $\gamma_{\text {Min }}$ of $24.24 \mathrm{mN} \mathrm{m}^{-1}$. This was in agreement with predictions based on earlier work of published mixed-bacterial spp. data, suggesting a fundamental limit to the ability of bacterial biosurfactants to reduce surface tensions in 
aqueous systems. This implies a biological restriction on the synthesis and export of these agents or a physical-chemical restriction on their functioning once produced.

\section{INTRODUCTION}

Biosurfactants are surface active chemicals expressed by a range of organisms that reduce liquid surface tensions $(\gamma)$ of aqueous and aqueous-hydrocarbon (oil) mixtures. Biosurfactants are widely used in biotechnology, including agriculture, cosmetics, food, pharmacology, bioremediation and oil recovery (see reviews by Ronsand Rosenberg, 2002; Mulligan, 2005; Marchant et al., 2012; Ławniczak et al., 2013; Sachdev and Cameotra, 2013), whilst the biological roles for bacterial biosurfactants include motility and virulence, the inhibition of nematode and protist predation, lysis of fungi and oomycetes, and the induction of systemic resistance in plants (reviewed by Raaijmakers et al., 2010), as well as modifying water distribution in soil pore networks (Fechtner et al., 2011).

Bacterial biosurfactant expression is readily surveyed using modifications of the drop-collapse assay (Persson and Molin, 1987), blood agar, oil plates and oil spays, and quantified by tensiometry of cultures or purified preparations (e.g. Bodour et al., 2003; Youssef et al., 2004; Burch et al., 2010). Surfactants can be behaviourally characterised by foaming, emulsion and oil-displacement assays, and are known to be structurally diverse, including glycolipids, lipopeptides, lipopolysaccharides, etc. (Marchant et al., 2012; Ławniczak et al., 2013; Sachdev and Cameotra, 2013).

The process from the isolation of biosurfactant expressing bacteria through to the chemical-structural analysis of biosurfactants is time-consuming, and a recent evaluation of the liquid surface tension reducing ability (LSTRA) of environmental pseudomonads by Individual distribution identification analysis suggests that biosurfactant activity may be limited $\left(\gamma_{\operatorname{Min}}\right)$ to $24 \mathrm{mN} \mathrm{m}^{-1}$ (Fechtner et al., 2011). The first bacterial biosurfactant to be characterised, surfactin, expressed by Bacillus subtilis, 
could reduce $\gamma$ to $27 \mathrm{mN} \mathrm{m}^{-1}$ (Peypoux et al. 1999). Perhaps surprisingly, since then significantly higher activities producing lower surface tensions have not been reported, despite the isolation and characterisation of many more biosurfactants from a range of different bacteria (e.g. $22-25 \mathrm{mN} \mathrm{m}^{-1}$ reported by Morikawa et al., 1993; Nielsen et al., 2002; Kuiper et al., 2004; Fechtner et al., 2011; Xie et al., 2011; Saimmai et al., 2012). A limit to biosurfactant activity suggests a biological restriction in the synthesis of these agents or a need to prevent self-damage during expression (Fechtner et al.,2011). In extremis, surfactant absorption to the air-liquid interface may be kinetically limited, and under these conditions the physical-chemical properties of the solution and atmosphere will also be important.

In this report, we sought to confirm $\gamma_{\text {Min }}$ by examining an independent set of pseudomonads recovered from soil and activated sludge, and a parallel set of recently published bacterial LSTRA measurements. Furthermore, we uncover substantial variation in biosurfactant behaviour within groups of isolates with very similar LSTRA, suggesting that these isolates are expressing a range of structurally-diverse biosurfactants.

\section{RESULTS AND DISCUSSION}

We obtained a diverse bacterial collection of 355 Pseudomonas or Pseudomonas-like isolates from activated sludge and soil samples by selection for aerobic growth on PSA-CFC plates. A preliminary analysis of isolate phenotype data by cluster analysis (after Robertson et al., 2013) demonstrated that the collection was diverse with few biological replicates. The collection was screened for LSTRA by drop-collapse assay of $18 \mathrm{~h}$ early stationary-phase KB cultures (after Persson and Molin, 1987), identifying 71 isolates $(20 \%$ of the total) likely to be expressing biosurfactants under the conditions used here. 
The positive isolates, plus 14 randomly-chosen drop-collapse-negative isolates,

were investigated further by quantitative tensiometry of cell-free $24 \mathrm{~h}$ stationary-phase shaken KB culture supernatants (Figure 1). Significant differences were found between isolates (ANOVA, $P<0.001$ ), with 57 LSTRA isolates found to significantly reduce the liquid surface tension of sterile $\mathrm{KB}$ from $53 \mathrm{mN} \mathrm{m}^{-1}$ and 28 that did not (the non-LSTRA group) (Dunnett's method, $\alpha=0.05$ ).

We note that 18 drop-collapse-positive isolates did not show significant LSTRA in culture supernatants whilst 4 drop-collapse-negative isolates did, indicating that the drop-collapse assay is not always reliable and that biosurfactant expression may be sensitive to growth conditions, as has been reported earlier (Burch et al., 2011; Fechtner et al., 2011). However, increasing incubation periods from $24 \mathrm{~h}$ to $48 \mathrm{~h}$ and maintaining cultures in the stationary phase for longer made little difference to final surface tensions, with only two of eight strains tested across the non-LSTRA, intermediate- $\gamma$ and low- $\gamma$ LSTRA groups showing significant but minor decreases in surface tensions of $2-5.3 \mathrm{mN} \mathrm{m}^{-1}$ (t-tests, $\left.\mathrm{P} \leq 0.05\right)$ (see Supplementary Table S1); more often surface tension increased by $0.3-4.1 \mathrm{mN} \mathrm{m}^{-1}(\mathrm{P} \leq 0.05)$, presumably due to the effects of culture aging and cellysis (Fechtner et al., 2011).

In order to predict the minimum bacterial biosurfactant activity $\left(\gamma_{\mathrm{Min}}\right)$ in cultures, we analysed the surface tension data for 50 LSTRA isolates that formed a single large homogeneous group (Isolates \#69 - \#67 as shown left to right in Figure 1), excluding the remaining 7 LSTRA isolates as they showed a poorer ability to reduce surface tension and appeared statistically to be an extension of the non-LSTRA group (TKHSD, $\alpha=0.05$ ). Individual distribution identification (IDI) analysis found that gamma, log-normal and log-logistic distributions fitted the surface tension data well, with the best-fit provided by a log-logistic distribution based on the Anderson-Darling goodness of fit test $(A D=0.497, P=0.293)$ predicting $\gamma_{\text {Min }}$ of $24.24 \mathrm{mN} \mathrm{m}^{-1}$ (Table 1). This compares well with predictions made from a collection of soil pseudomonads (Fechtner 
et al., 2011) and from a random sample of published reports of LSTRA from a range of bacteria including non-pseudomonads (Table 1; see also Supplementary Table S2 and references therein). We are not aware of any bacterium able to reduce the liquid surface tension of cultures significantly below $22-25 \mathrm{mN} \mathrm{m}^{-1}$ (Morikawa et al., 1993; Nielsen et al., 2002; Kuiper et al., 2004; Fechtner et al., 2011; Xia et al., 2011; Saimmai et al., 2012) and the theoretical $24 \mathrm{mN} \mathrm{m}^{-1}$ limit identified by Fechtner et al. (2011) and confirmed by the work reported here (we note that the lowest value of 22.56 $\mathrm{mN} \mathrm{m}^{-1}$ given by Xia et al. (2011) is reported as a single measurement with no indication of reproducibility). Although biosurfactant activity is concentration, temperature and solution dependant, we do not believe that the theoretical limit determined here is unique to KB culture supernatants, as other reports of biosurfactant surface tensions in the $22-25 \mathrm{mN} \mathrm{m}^{-1}$ range have been for a variety of spent culture media and solutions of purified surfactant. It is important to note that in bacterial cultures the critical micelle concentration, (CMC), corresponding to the lowest surface tension produced by a biosurfactant, may not be achieved for a number of reasons, including growth limitations and biosurfactant interactions with other compounds. Further research is necessary to determine whether the theoretical limit is the same for purified biosurfactant solutions; if it is, then we would suggest that there is a biological restriction on the expression of more active biosurfactants, but if not, we would suggest that the limit is at least partially restricted by the biochemical system in which biosurfactants and other compounds interact.

We were interested in determining whether there is evidence of structural diversity amongst the biosurfactants produced by the low- $\gamma$ LSTRA isolates, as this could be used to select isolates for further chemical-structural analyses and testing of novel biosurfactants. We approached this by identifying two homogeneous groups of isolates producing significant, but small differences in surface tensions $\square \square$ Group I: 25.2 $\square \square \square 0.1 \mathrm{mN} \mathrm{m}^{-1} \square \square \square \square \square$ Group II: $26.7 \square \square \square 0.5 \mathrm{mN} \mathrm{m}^{-1}$; TK-HSD, $\left.\alpha=0.05\right)$. We 
then investigated the biosurfactant behaviours of these isolates using emulsion, foaming and oil-displacement assays (see Table 2 listing the behaviours of these and other low- $\gamma$ LSTRA isolates). Cluster analysis of the behaviour data showed significant variation between isolates, suggesting that there may be structural diversity in the biosurfactants they express (Figure 2). This analysis failed to cluster the isolates into the original set of two groups, indicating that both groups were likely to be producing a similar set of diverse biosurfactants. The difference between Group I and tensions cannot be explained by distinct types of biosurfactant, as surface tension and behaviour were not significantly associated (Fisher's exact test, $P=0.1201$ ). In contrast, surface tension and isolate phenotype (determined using a number of enzymatic and growth-based assays) were weakly associated $(R=0.0768)$, suggesting that closely-related isolates may express more similar biosurfactant types than distantly-related isolates.

\section{CONCLUDING STATEMENT}

Surveying environmental bacterial isolates for the expression of biosurfactants is relatively straight forward using a series of techniques such as the drop collapse assay, blood agar, oil plates and oil sprays, followed by the quantification of surface tension by tensiometry. However, there appears to be a fundamental limit to the reduction of liquid surface tension of bacterial cultures of $24 \mathrm{mN} \mathrm{m}^{-1}$, suggesting that the hunt for more active agents will become progressively less rewarding. Nonetheless, within very narrow ranges of liquid surface tension, there is evidence for bacterial biosurfactants with substantially different behaviours that may be of greater interest in biotechnology applications than the absolute surface tension that can be achieved.

\section{MATERIALS AND METHODS}


Bacteria were isolated from activated sludge samples from the Hatton Wastewater Treatment Plant at Arbroath and soil samples from a roadside site near Dundee with a history of hydrocarbon contamination by growth on Pseudomonas-selective agar plates (PSA-CFC, Oxoid, UK) under aerobic conditions for $72 \mathrm{~h}$ at $20^{\circ} \mathrm{C}$. Randomly-chosen colonies were re-streaked on PAS-CFC plates before being used to inoculate overnight KB shaken cultures (King's $\mathrm{B} ; 10 \mathrm{~g}$ glycerol, $1.5 \mathrm{~g} \mathrm{~K}_{2} \mathrm{HPO}_{4}, 1.5 \mathrm{~g} \mathrm{MgSO} 4.7 \mathrm{H}_{2} \mathrm{O}$ and $20 \mathrm{~g}$ Proteose peptone No. 3 (Becton, Dickinson and Company, UK) per litre) and aliquots of these maintained at $-80^{\circ} \mathrm{C}$ as $15 \%(\mathrm{v} / \mathrm{v})$ glycerol stocks. Isolates were phenotypically characterized using a number of enzymatic and growth-based assays after Robertson et al. (2013) (see Supplementary Information for further details), and a drop collapse assay (Persson and Molin, 1987) used to screen isolates for those expressing biosurfactants in $18 \mathrm{~h}$ early-stationary-phase shaken KB cultures.

The liquid surface tension $(\gamma)$ of replicate cell-free shaken $24 \mathrm{~h}$ stationary-phase shaken KB culture supernatants ( $n=4)$ were quantitatively measured using a K100 Mk 2 Tensiometer (Krüss, Germany) by the rod method at $20^{\circ} \mathrm{C}$ as described by Fechtner et al. (2011) (using this methôd, $y_{\text {water }}$ was $73.2 \pm 0.1 \mathrm{mN} \mathrm{m}^{-1}$ ). Data were examined by t-tests, ANOVA, and post hoc multiple comparison tests including Dunnett's method with a control and Tukey-Kramer HSD (TK-HSD) (JMP 7, SAS Institute, USA). Individual distribution identification (IDI) analysis based on the Anderson-Darling (AD) goodness of fit test was used to identify theoretical probability distributions to fit surface tension data and predict $\gamma_{\text {Min }}$ using the threshold parameters of the fitted distributions (MINITAB v.15, Minitab Ltd, UK). Published surface tension data for 59 mixed bacterial spp. was also analysed by IDI (see Supplementary Information for further details).

Biosurfactant behaviour was assessed by foaming assay (after Sathe and Salunke, 1981), and emulsion and oil-displacement assays (Youssef et al., 2004; Prieto et al., 2008) (see Supplementary Information for further details). Biosurfactant behaviours were investigated by pairwise correlations $\left(\mathrm{r}^{2}\right)$ and cluster analysis using a 
214 hierarchical approach and Ward's minimum variance method (JMP 7). The association 215 between surface tension, phenotype and surfactant behaviour data was assessed

\section{ACKNOWLEDGEMENTS}

We thank Bob Peter and Alison Duffy of the Urban Water Technology Centre at Abertay University for their help in obtaining samples from the Hatton Wastewater Treatment Plant and the SUDS site. IM is a PhD student of AS funded by the Nigerian Petroleum Technology Development Fund. SM and AS are also members of the Scottish Alliance for Geoscience Environment and Society (SAGES).

\section{CONFLICT OF INTEREST}

No conflict of interest declared.

\section{SUPPORTING INFORMATION}

- Materials and methods for the emulsion, foaming and oil-displacement assays, including the selection of published reports of bacterial surface tension measurements and the association analysis.

List of phenotype assays used in this work.

- Table S1: Differences in liquid surface tension at $24 \mathrm{~h}$ and $48 \mathrm{~h}$.

- Table S2: Bacterial liquid surface tension reducing activity (LSTRA).

- References for Supplementary Table S2. 


\section{REFERENCES}

Burch, A.Y., Shimada, B.K., Browne, P.J. and Lindow, S.E. (2010) Novel highthroughput detection method to assess bacterial surfactant production. Appl Environ Microbiol 76, 5363-5372.

Chen, C-Y., Baker, S.C. and Darton, R.C. (2007) The application of a high throughput analysis method for the screening of potential biosurfactants from natural sources. $J$ Microbiol Methods 70, 503-510.

Fechtner, J., Koza, A., Dello Sterpaio, P., Hapca, S.M. and Spiers, A.J. (2011) Surfactants expressed by soil pseudomonads alter local soil-water distribution suggesting a hydrological role for these compounds. FEMS Microbiology Ecology 78, $50-58$.

Kuiper, I., Lagendijk, E.L., Pickford, R., Derrick, J.P., Lamers, G.E.M., Thomas-Oates, J.E., Lugtenberg, B.J.J. and Bloemberg, G.V. (2004) Characterization of two Pseudomonas putida lipopeptide biosurfactants, putisolvin I and II, which inhibit biofilm formation and break down existing biofilms. Mol Microbiol 51, 97-113.

Ławniczak, Ł. Marecik, R. and Chrzanowski, Ł. (2013) Contributions of biosurfactants to natural or induced bioremediation. Appl Microbiol Biotechnol 97, 2327-2339.

Marchant, R. and Banat, I.M. (2012) Microbial biosurfactants: challenges and opportunities for future exploitation. Trends Biotechno/ 30, 558-565.

Morikawa, M., Daido, H., Takao, T., Murata, S., Shimonishi, Y. and Imanaka, T. (1993) A new lipopeptide biosurfactant produced by Arthobacter sp. Strain MIS38. J Bacteriol $175,6459-6466$. 
Mulligan, C.N. (2005) Environmental applications for biosurfactants. Environ Pollution 133, 183-198.

Nielsen, T.H., Sørensen, D., Tobiasen, C., Andersen, J.B., Christophersen, C.,

Givskov, M. and Sørensen, J. (2002) Antibiotic and biosurfactant properties of cyclic lipopeptides produced by fluorescent Pseudomonas spp. from the Sugar beet rhizosphere. Appl Environ Microbiol 68, 3416-3423.

Persson, A. and Molin, G. (1987) Capacity for biosurfactant production of environmental Pseudomonas and Vibrionaceae growing on carbohydrates. Appl Microbiol Biotechnol 26, 439-442.

Peypoux, F., Bonmatin, J.M. and Wallach, J. (1999) Recent trends in the biochemistry of surfactin. Appl Microbiol Biotechnol 51, 553-563.

Prieto, L.M., Michelon, M. Burkert, J.F.M., Kalil, S.J. and Burkert, C.A.V. (2008) The production of rhamnolipid by a Pseudomonas aeruginosa strain isolated from a southern coastal zone in Brazil. Chemosphere 71, 1781-1785.

Raaijmakers, J.M., De Bruijn, I., Nybroe, O. and Ongena, M. (2010) Natural functions of lipopeptides from Bacillus and Pseudomonas: more than surfactants and antibiotics. FEMS Microbiol Rev 34, 1037-1062.

Robertson, M., Hapca, S.M., Moshynets, O. and Spiers, A.J. (2013) Air-liquid interface biofilm formation by psychrotrophic pseudomonads recovered from spoilt meat. Antonie van Leeuwenhoek 103, 251-259. 
Ron, E.Z. and Rosenberg, E. (2002) Biosurfactants and oil bioremediation. Curr Opin Biotechnol 13, 249-252.

Sachdev, D.P. and Cameotra, S.S. (2013) Biosurfactants in agriculture. Appl Microbiol Biotechnol 97, 1005-1016.

Saimmai, A., Rukadee, O., Onlamool, T., Sobhon, V. and Maneerat, S. (2012) Isolation and functional characterization of a biosurfactant produced by a new and promising strain of Oleomonas sagaranensis AT18. World J Microbiol Biotechnól28, 2973-2986.

Sathe, S.K. and Salunkhe, D.K. (1981) Functional properties of the great northern bean (Phaseolus vulgaris L.) proteins: Emulsion, foaming, viscosity, and gelation properties. J Food Sci 46, 71-81.

Xia, W-J., Dong, H-P., Yu, L. and Yu, D-F.(2011) Comparative study of biosurfactant produced by microorganisms isolated from formation water of petroleum reservoir. Colloids Surf A: Physicochem Eng Aspects 392, 124-130.

Youssef, N.H., Duncan, K.E., Nagle, D.P., Savage, K.N., Knapp, R.M. and Mclnerney, M.J. (2004) Comparison of methods to detect biosurfactant production by diverse microorganisms. J Microbiol Methods 56, 339-347.

Zhang, X., Xu, D., Zhu, C., Lundaa, T. and Kerstin, E.S. (2012) isolation and identification of biosurfactant producing and crude oil degrading Pseudomonas aeruginosa strains. Chemical Eng J 209, 138-146. 


\section{FIGURE LEGENDS}

Figure 1. Contaminated soil and activated sludge-isolated pseudomonads express liquid surface tension-reducing activity. A total of 57 of 85 isolates significantly reduced the liquid surface tension $\left(\gamma_{\square}\right)$ of sterile King's $\mathrm{B}(\mathrm{KB})$ medium from $52.8 \pm 0.5 \mathrm{mN} \cdot \mathrm{m}^{-1}$ (dashed line) to $24.5 \pm 0.1-49.1 \pm$ $1.4 \mathrm{mN} \mathrm{m}^{-1}$, whilst the remaining 28 isolates did not (Dunnett's method using $\gamma_{\square \square}$ as the control, $\alpha=0.05$ ) (left to right, LSTRA isolates \#69-\#75 and non-LSTRA isolates \#114 - \#311, respectively). The LSTRA isolates are further differentiated into the low and intermediate- $\gamma$ groups as indicated. Liquid surface tension was determined by tensiometry of cell-free supernatants produced from $24 \mathrm{~h}$ stationary-phase shaken KB culture cultures. Mean and standard errors $(n=4)$ are shown. The bimodal distribution of surface tension measurements is shown in the inset histogram.

Figure 2. The low- $\gamma$ LSTRA isolates show evidence of variation in biosurfactant behaviour. Cluster analysis of the biosurfactant behaviour data for the two homogeneous groups of isolates producing different surface tensions (Group I, circles; and II, squares) demonstrates significant variation between isolates and an overlap (mixing) of the two groups. Biosurfactant behaviours were determined by foaming, emulsion and oil-displacement assays (see Table 2), and isolates are linked on the basis of similarity. The dendrogram is shown with an even-spacing horizontal scale. 
TABLES

Table 1. A comparison of predicted $\gamma_{\text {Min }}$ determined by IDI analysis.

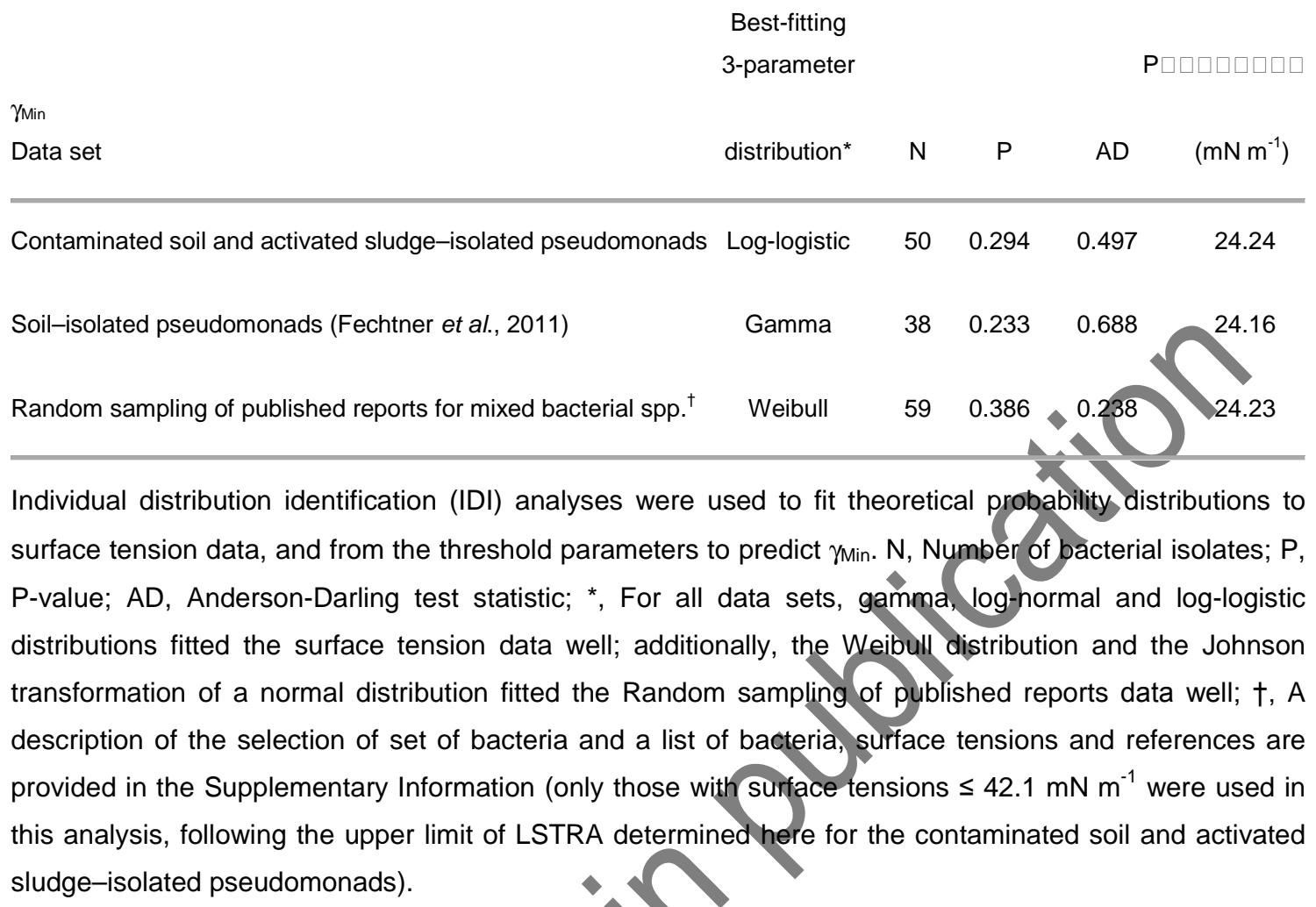

Individual distribution identification (IDI) analyses were used to fit theoretical probability distributions to surface tension data, and from the threshold parameters to predict $\gamma_{\text {Min }}$. N, Number of bacterial isolates; $P$, P-value; AD, Anderson-Darling test statistic; *, For all data sets, gamma, log-normal and log-logistic distributions fitted the surface tension data well; additionally, the Weibull distribution and the Johnson transformation of a normal distribution fitted the Random sampling of published reports data well; $\dagger, \mathrm{A}$ description of the selection of set of bacteria and a list of bacteria, surface tensions and references are provided in the Supplementary Information (only those with Surface tensions $\leq 42.1 \mathrm{mN} \mathrm{m}^{-1}$ were used in this analysis, following the upper limit of LSTRA determined here for the contaminated soil and activated sludge-isolated pseudomonads).

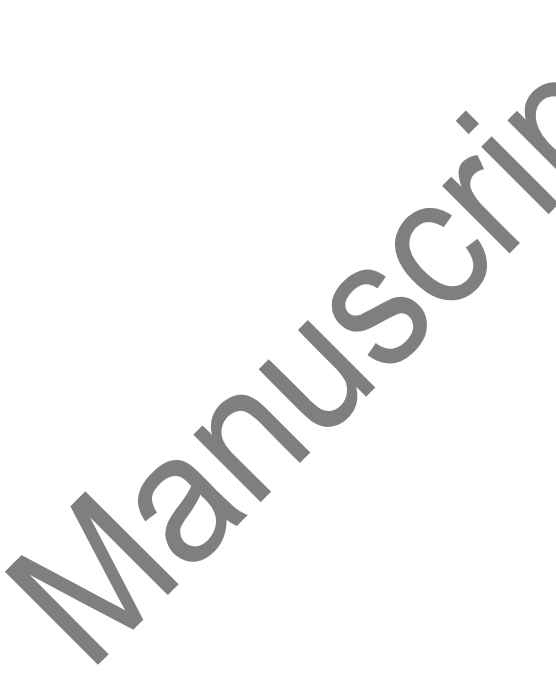


Table 2. Biosurfactant behaviours of low- $\gamma$ LSTRA isolates.

\begin{tabular}{|c|c|c|c|c|c|c|}
\hline \multirow[b]{2}{*}{ Isolate } & \multirow{2}{*}{$\begin{array}{l}\text { Liquid surface } \\
\text { tension } \\
\left(\mathrm{mN} \mathrm{m}^{-1}\right)\end{array}$} & \multicolumn{3}{|c|}{ Emulsion Assay } & \multirow{2}{*}{$\begin{array}{l}\text { Foam } \\
\text { stabilitity } \\
(\%)\end{array}$} & \multirow{2}{*}{$\begin{array}{l}\text { Oil- } \\
\text { displacement }{ }^{d} \\
(\mathrm{~mm})\end{array}$} \\
\hline & & $\mathrm{O}_{\mathrm{i}}$ & $A_{i}$ & $E_{i}$ & & \\
\hline 69 & $24.5 \pm 0.1$ & $0.4 \pm 0.0$ & $0.3 \pm 0.0$ & $0.3 \pm 0.0$ & $0.1 \pm 0.1$ & $30.0 \pm 0.0$ \\
\hline 83 & $24.8 \pm 0.2$ & $0.1 \pm 0.0$ & $0.3 \pm 0.0$ & $0.6 \pm 0.0$ & $0.2 \pm 0.0$ & $39.7 \pm 2.0$ \\
\hline 101 & $24.9 \pm 0.1$ & $0.0 \pm 0.0$ & $0.3 \pm 0.0$ & $0.7 \pm 0.0$ & $0.1 \pm 0.1$ & $10.0 \pm 0.6$ \\
\hline 303 & $25.1 \pm 0.0$ & $0.1 \pm 0.0$ & $0.1 \pm 0.1$ & $0.7 \pm 0.1$ & $0.1 \pm 0.1$ & $30.0 \pm 0.0$ \\
\hline 190 & $25.1 \pm 0.1$ & $0.1 \pm 0.1$ & $0.4 \pm 0.0$ & $0.5 \pm 0.1$ & $0.2 \pm 0.0$ & $24.3 \pm 0.7$ \\
\hline 152 & $25.1 \pm 0.0$ & $0.1 \pm 0.0$ & $0.2 \pm 0.0$ & $0.6 \pm 0.0$ & $0.2 \pm 0.1$ & $30.0 \pm 2.9$ \\
\hline 178 & $25.2 \pm 0.4$ & $0.2 \pm 0.0$ & $0.3 \pm 0.0$ & $0.4 \pm 0.1$ & $0.2 \pm 0.0$ & $27.7 \pm 1.2$ \\
\hline 6 & $25.2 \pm 0.2$ & $0.1 \pm 0.0$ & $0.3 \pm 0.0$ & $0.6 \pm 0.0$ & $0.5 \pm 0.0$ & $31.0 \pm 0.6$ \\
\hline 1 & $25.3 \pm 0.1$ & $0.1 \pm 0.0$ & $0.3 \pm 0.0$ & $0.6 \pm 0.0$ & $0.2 \pm 0.0$ & $34.0 \pm 1.5$ \\
\hline 65 & $25.3 \pm 0.2$ & $0.2 \pm 0.1$ & $0.1 \pm 0.1$ & $0.7 \pm 0.0$ & $0.3 \pm 0.1$ & $46.3 \pm 1.9$ \\
\hline 211 & $25.3 \pm 0.1$ & $0.1 \pm 0.0$ & $0.3 \pm 0.0$ & $0.6 \pm 0.0$ & $0.2 \pm 0.0$ & $41,3 \pm 1.5$ \\
\hline 194 & $25.4 \pm 0.1$ & $0.1 \pm 0.0$ & $0.3 \pm 0.0$ & $0.6 \pm 0.0$ & $0.2 \pm 0.0$ & 0. \\
\hline 79 & $25.5 \pm 0.1$ & $0.1 \pm 0.1$ & $0.2 \pm 0.0$ & $0.6 \pm 0.1$ & $0.3 \pm 0.0$ & $8.3 \pm 0.9$ \\
\hline 179 & $25.5 \pm 0.1$ & $0.2 \pm 0.0$ & $0.3 \pm 0.0$ & $0.5 \pm 0.0$ & $0.2 \pm 0.0$ & $8.3 \neq 0.9$ \\
\hline 86 & $25.5 \pm 0.1$ & $0.5 \pm 0.1$ & $0.0 \pm 0.0$ & $0.6 \pm 0.1$ & $0.2 \pm 0.0$ & $44.0 \pm 1.5$ \\
\hline 97 & $25.7 \pm 0.1$ & $0.3 \pm 0.1$ & $0.2 \pm 0.1$ & $0.6 \pm 0.0$ & $0.2 \pm 0.1$ & $29.0 \pm 0.6$ \\
\hline 2 & $25.9 \pm 0.1$ & $0.2 \pm 0.0$ & $0.3 \pm 0.0$ & $0.6 \pm 0.0$ & $0.3 \pm 0.1$ & $13.0 \pm 0.6$ \\
\hline 236 & $26.4 \pm 0.1$ & $0.1 \pm 0.0$ & $0.3 \pm 0.0$ & $0.6 \pm 0.0$ & $0.5 \pm 0.0$ & $42.7 \pm 2.3$ \\
\hline 197 & $26.4 \pm 0.0$ & $0.1 \pm 0.0$ & $0.3 \pm 0.0$ & $0.7 \pm 0.0$ & $0.3 \pm 0.0$ & $10.0 \pm 0.6$ \\
\hline 169 & $26.4 \pm 0.1$ & $0.1 \pm 0.0$ & $0.3 \pm 0.0$ & $0.6 \pm 0.0$ & $0.4 \pm 0.1$ & $30.7 \pm 1.8$ \\
\hline 88 & $26.5 \pm 0.1$ & $0.1 \pm 0.0$ & $0.2 \pm 0.0$ & $0.7 \pm 0.0$ & $1.0 \pm 0.0$ & $8.0 \pm 0.6$ \\
\hline 239 & $26.6 \pm 0.1$ & $0.2 \pm 0.0$ & $0.3 \pm 0.0$ & $0.5 \pm 0.1$ & $0.2 \pm 0.1$ & $29.7 \pm 0.3$ \\
\hline 175 & $26.7 \pm 0.2$ & $0.2 \pm 0.0$ & $0.3 \pm 0.0$ & $0.7 \pm 0.0$ & $0.2 \pm 0.1$ & $2.3 \pm 0.3$ \\
\hline 166 & $26.7 \pm 0.2$ & $0.2 \pm 0.0$ & $0.3 \pm 0.0$ & $0.6 \pm 0.0$ & $0.3 \pm 0.1$ & $21.0 \pm 0.6$ \\
\hline 335 & $26.7 \pm 0.1$ & $0.1 \pm 0.0$ & $0.3 \pm 0.0$ & $0.7 \pm 0.0$ & $0.1 \pm 0.1$ & $3.3 \pm 0.3$ \\
\hline 336 & $26.7 \pm 0.1$ & $0.1 \pm 0.0$ & $0.3 \pm 0.0$ & $0.6 \pm 0.0$ & $0.2 \pm 0.1$ & $43.7 \pm 0.9$ \\
\hline 355 & $26.8 \pm 0.1$ & $0.4 \pm 0.0$ & $0.4 \pm 0.0$ & $0.2 \pm 0.0$ & $0.4 \pm 0.1$ & $37.3 \pm 1.5$ \\
\hline 105 & $26.8 \pm 0.1$ & $0.1 \pm 0.0$ & $0.3 \neq 0.0$ & $0.7 \pm 0.0$ & $0.2 \pm 0.0$ & $15.3 \pm 0.3$ \\
\hline 107 & $26.9 \pm 0.1$ & $0.1 \pm 0.0$ & 0.2 & $0.7 \pm 0.0$ & $0.3 \pm 0.0$ & $1.0 \pm 0.6$ \\
\hline 111 & $26.9 \pm 0.2$ & $0.0 \pm 0.0$ & $0.3 \pm 0.0$ & $0.7 \pm 0.0$ & $0.4 \pm 0.1$ & $8.0 \pm 0.6$ \\
\hline 94 & $27.2 \pm 0.2$ & $0.2 \pm 0.1$ & $0.1 \pm 0.1$ & $0.7 \pm 0.0$ & $0.2 \pm 0.0$ & $8.0 \pm 0.6$ \\
\hline 354 & $27.3 \pm 0.0$ & $0.1 \pm 0.0$ & $0.3 \pm 0.0$ & $0.6 \pm 0.0$ & $0.2 \pm 0.1$ & $34.3 \pm 2.8$ \\
\hline 195 & $27.8 \pm 0.4$ & $0.1 \pm 0$ & $0.2 \pm 0.1$ & $0.7 \pm 0.0$ & $0.2 \pm 0.1$ & $2.3 \pm 0.3$ \\
\hline 327 & $27.9 \pm 0.2$ & $0.1 \pm$ & $0.3 \pm 0.0$ & $0.6 \pm 0.1$ & $0.4 \pm 0.1$ & $1.0 \pm 0.0$ \\
\hline 106 & $28.4 \pm 0.1$ & $0.2 \pm 0.0$ & $0.2 \pm 0.0$ & $0.6 \pm 0.1$ & $0.2 \pm 0.0$ & $11.0 \pm 0.6$ \\
\hline 9 & $28.6 \pm 0.0$ & $0.2 \pm 0.0$ & $0.3 \pm 0.0$ & $0.6 \pm 0.0$ & $0.1 \pm 0.0$ & $20.7 \pm 1.2$ \\
\hline 8 & $28.6 \pm 0.2$ & $0.1 \pm 0.0$ & $0.2 \pm 0.0$ & $0.7 \pm 0.0$ & $0.2 \pm 0.0$ & $14.0 \pm 0.6$ \\
\hline 85 & $28.9 \pm 0.1$ & $0.3 \pm 0.0$ & $0.1 \pm 0.1$ & $0.6 \pm 0.1$ & $0.2 \pm 0.1$ & $10.7 \pm 0.7$ \\
\hline 63 & 28.9 & $0.5 \pm 0.0$ & $0.3 \pm 0.0$ & $0.2 \pm 0.0$ & $0.3 \pm 0.1$ & $34.0 \pm 0.6$ \\
\hline 342 & & $0.0 \pm 0.0$ & $0.2 \pm 0.0$ & $0.7 \pm 0.0$ & $0.2 \pm 0.0$ & $2.0 \pm 0.0$ \\
\hline 183 & & $0.1 \pm 0.0$ & $0.2 \pm 0.1$ & $0.7 \pm 0.1$ & $0.2 \pm 0.0$ & $43.0 \pm 0.6$ \\
\hline
\end{tabular}

418

419 The surfactant behaviours of the first 41 low- $\gamma$ LSTRA isolates were determined and listed here in increasing surface tension order (as shown in Fig. 1). Means 420 and standard errors are shown. a, Liquid surface tensions were determined by tensiometry of cell-free $18 \mathrm{~h} \mathrm{~KB}$ cultures $(n=4)$; b, Oil $\left(\mathrm{O}_{i}\right)$, aqueous $\left(\mathrm{A}_{i}\right)$ and

emulsion $\left(E_{i}\right)$ indices were determined as the relative height of each layer after shaking a mixture of $18 \mathrm{~h} \mathrm{~KB}$ culture and oil after $24 \mathrm{~h}(n=3)$; c, Foam stability was determined as the percentage reduction of foam heights after $2 \mathrm{~h}$ using $18 \mathrm{~h} \mathrm{~KB}$ cultures $(n=3)$; d, The displacement of an oil film by a drop of $18 \mathrm{~h}$ KB culture was measured as the drop diameter $(\mathrm{mm})$ after $5 \mathrm{~s}(n=3)$. Significant correlations were observed between $\mathrm{A}_{\mathrm{i}} x \mathrm{E}_{\mathrm{i}}\left(\mathrm{r}^{2}=-0.4510, \mathrm{P}=0.0031\right), \mathrm{O}_{\mathrm{i}} x \mathrm{E}_{\mathrm{i}}\left(\mathrm{r}^{2}=\right.$ -0.7295, $\mathrm{P}<0.0001)$, Oil-displacement $x \mathrm{E}_{\mathrm{i}}\left(\mathrm{r}^{2}=-0.3368, \mathrm{P}=0.0313\right)$ and Oil-displacement $x \mathrm{O}_{\mathrm{i}}\left(\mathrm{r}^{2}=0.3867, \mathrm{P}=0.0125\right)$; all other pair-wise correlations were not significant $(P \leq 0.05)$. 


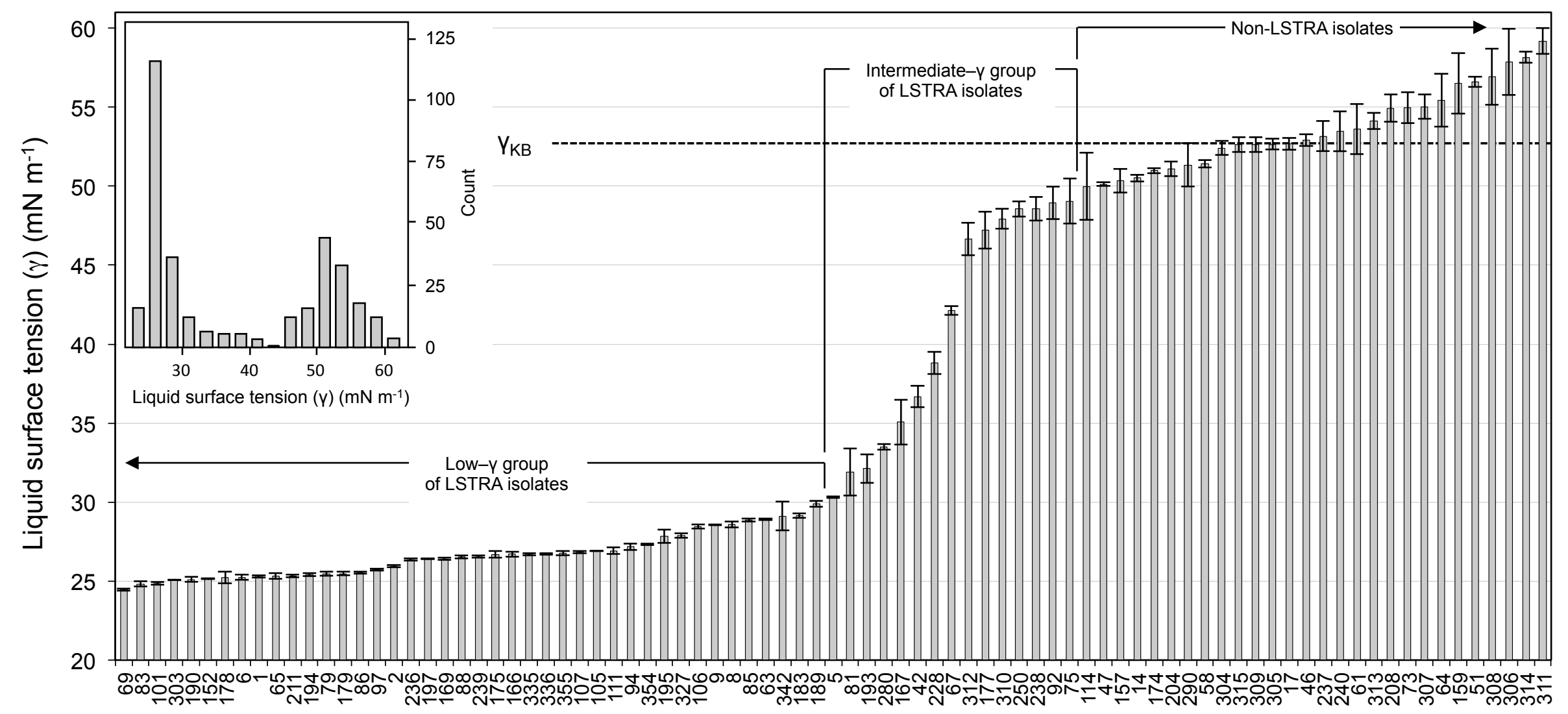

Contaminated soil and activated sludge-isolated pseudomonads

Figure 1 


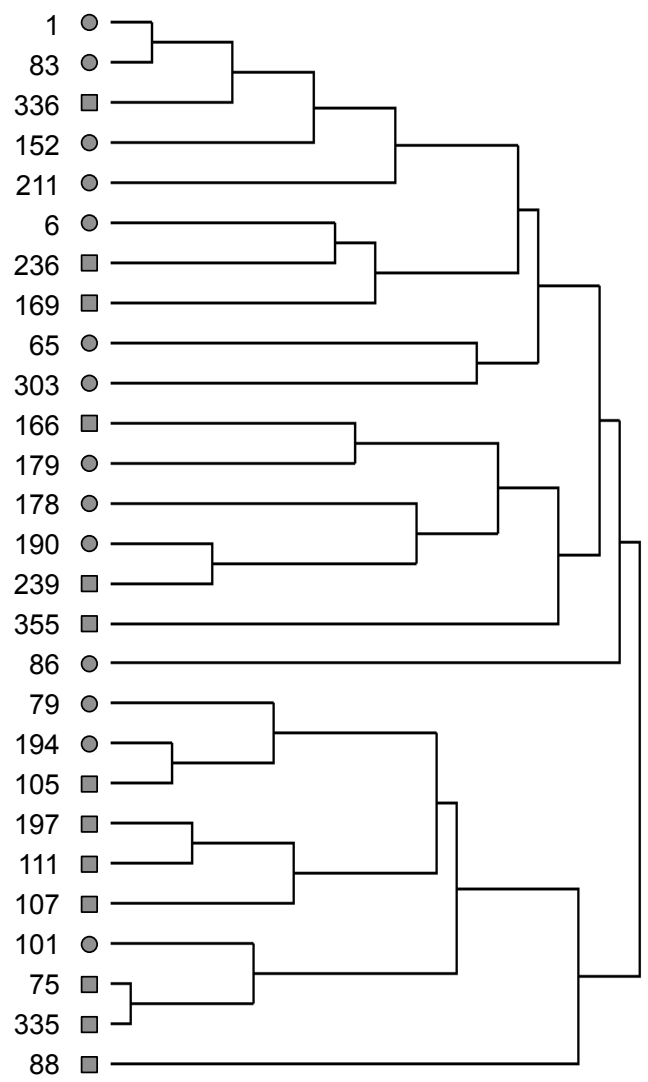

Figure 2 